\title{
Conversion Matrix Analysis of GaAs HEMT Active Gilbert Cell Mixers
}

\author{
Jiang, Chenhui; Johansen, Tom Keinicke; Krozer, Viktor
}

Published in:

Proceedings of the Integrated Nonlinear Microwave and Millimetre-wave Circuits (INMMiC)

Link to article, DOI:

10.1109/INMMIC.2006.283518

Publication date:

2006

Document Version

Publisher's PDF, also known as Version of record

Link back to DTU Orbit

Citation (APA):

Jiang, C., Johansen, T. K., \& Krozer, V. (2006). Conversion Matrix Analysis of GaAs HEMT Active Gilbert Cell Mixers. In Proceedings of the Integrated Nonlinear Microwave and Millimetre-wave Circuits (INMMiC) (pp. 9497). IEEE. https://doi.org/10.1109/INMMIC.2006.283518

\section{General rights}

Copyright and moral rights for the publications made accessible in the public portal are retained by the authors and/or other copyright owners and it is a condition of accessing publications that users recognise and abide by the legal requirements associated with these rights.

- Users may download and print one copy of any publication from the public portal for the purpose of private study or research.

- You may not further distribute the material or use it for any profit-making activity or commercial gain

- You may freely distribute the URL identifying the publication in the public portal 


\title{
Conversion Matrix Analysis of GaAs HEMT Active Gilbert Cell Mixers
}

\author{
Chenhui Jiang, Tom K. Johanson, and Viktor Krozer \\ Department of Electromagnetic System, Ørsted \\ Technical Univeristy of Denmark \\ DK2800 Lyngby - Denmark
}

\begin{abstract}
In this paper, the nonlinear model of the GaAs HEMT active Gilbert cell mixer is investigated. Based on the model, the conversion gain expression of active Gilbert cell mixers is derived theoretically by using conversion matrix analysis method. The expression is verified by harmonic balance simulation with Angelov HEMT model in Agilent Advanced Design System (ADS) and by chip measurement results.
\end{abstract}

Index Terms-Active Gilbert cell mixers, Conversion gain, Conversion matrix analysis, GaAs HEMT.

\section{INTRODUCTION}

In radar and wireless communication systems, the mixer plays a very important role in the way of providing frequency conversion between different frequency bands. The active Gilbert cell mixer [1] shown in Fig. 1 (a) is now widely used in this application. In the mixer, T1-T4 compose a switching quad, and T5-T6 represents the transconductance stage. The main virtues for this topology are its high conversion gain in a broad frequency range and excellent port-to-port isolation. In order to investigate the performance of the active mixer for wideband application, a theoretical analysis on the main bandwidth limitations in the Gilbert cell topology is required. When the large LO signals are fed to the switching quad of the Gilbert cell, the switching quad can be represented by a linear time-varying circuit. Therefore, a traditional linear time-invariant analysis on such circuit becomes invalid. Instead, conversion matrix analysis [2] is applied in order to obtain an analytical expression for the conversion gain as a function of the RF input frequency and IF output frequency. This paper describes a theoretical analysis of the conversion gain of GaAs HEMT active mixers based on the Gilbert cell topology. An analytical expression is derived which is capable of predicting the main bandwidth limitations for active mixers performing frequency conversion of wideband signals. The analysis is verified by harmonic balance simulations in Agilent Advanced Design System (ADS) using the nonlinear HEMT Angelov model. This paper presents an extension of the

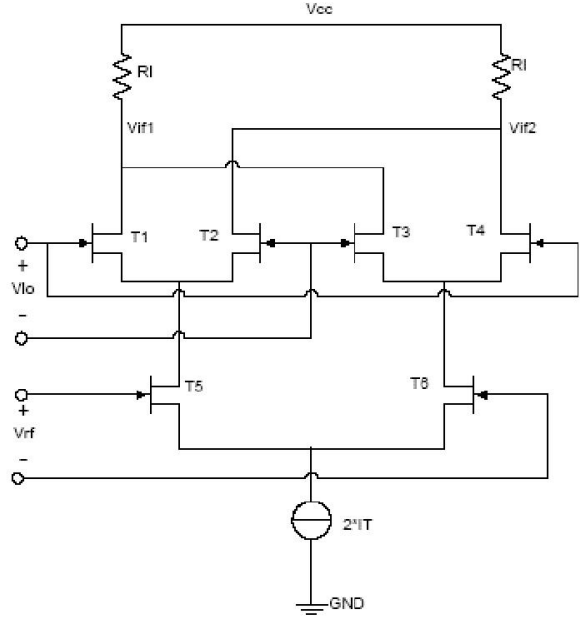

Fig. 1. The GaAs HEMT Active Gilbert cell mixer

analysis by the authors on HBT device to include HEMT device $[3,4]$.

\section{CONVErsion Matrix ANALysis}

Conversion matrix analysis is a powerful tool for solving problems where a nonlinear device is driven by a single large sinusoidal signal and a much smaller signal. It is a suitable technique for analyzing and designing mixers. First, nonlinear devices driven by large signals are analyzed by the harmonic-balance method. The nonlinear elements in the device's equivalent circuit are then linearized to create small-signal linear time-varying elements. Finally, a small-signal analysis can be performed on the time-varying circuit.

Fig. 2 shows the time-varying small-signal equivalent circuit model of the half Gilbert cell mixer. The switching quad is composed of time-varying elements, and the transconductance stage is composed of $I_{s}$ and $Z_{d s}$. The switching quad driven by the large LO signal is suitable for the conversion matrix analysis, and the transconductance stage driven by small RF 


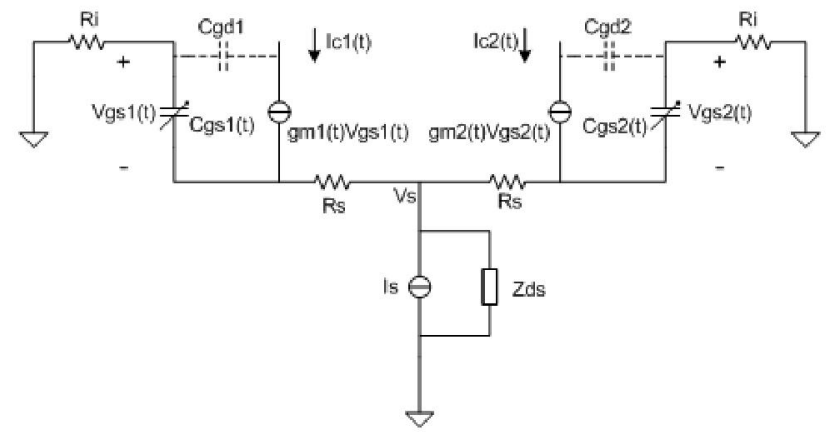

Fig. 2. The small-signal linear time-varying equivalent circuit model for GaAs HMET active Gilbert cell mixers

signal is suitable for the time-invariant linear analysis.

\section{A. Switch quad analysis}

According to the behavior of the mixer, the time-varying elements in the Gilbert cell mixer excited by the small RF signal result in mixing frequencies. These frequencies can be represented as

$$
\omega_{n}=\omega_{i f}+n \omega_{L O} \quad n=0, \pm 1, \pm 2, \pm 3, \ldots \ldots .
$$

where $\omega_{i f}=\left|\omega_{L O}-\omega_{r f}\right|$ is the IF angular frequency, $\omega_{r f}$ and $\omega_{L O}$ are the RF and LO angular frequency respectively. The frequencies interested can be expressed by

$$
\omega_{n}=\left\{\begin{array}{ccc}
\omega_{i f} & \text { for } & n=0 \\
\omega_{r f} & \text { for } & n=1 \\
2 \omega_{i f}-\omega_{r f} & \text { for } & n=-1
\end{array}\right.
$$

Because the switch quad is excited by the large LO signal, the time-varying nonlinear transconductance of transistors in the switch quad can be expressed by

$$
g_{m}(t)=\left\{\begin{array}{cc}
G_{\max } & \text { for }\left|\omega_{L O} t-2 n \pi\right|<(\pi-\alpha) / 2 \\
0 & \text { else }
\end{array}\right.
$$

where the $G_{\max }$ is the maximum transconductance of transistors in the switching quad and $\boldsymbol{\alpha}$ is the conduction angle in one period. In order to perform the conversion matrix calculation, the Fourier coefficient of the transconductance should be derived to compose the related conversion matrix. These are given by

$$
G_{n}=\left\{\begin{array}{lll}
\frac{\alpha}{\pi} G_{\max } & \text { for } & n=0 \\
\frac{\sin \alpha}{\pi} G_{\max } & \text { for } & n= \pm 1 \\
\frac{\sin 2 \alpha}{2 \pi} G_{\max } & \text { for } & n= \pm 2
\end{array}\right.
$$

Normally, $\boldsymbol{\alpha}$ is equal to $\pi / 2$ because the duty cycle is considered ideal here. So, we can obtain the conversion matrix of the transconductance given by

$$
\underline{\underline{G}}_{m}=\left[\begin{array}{ccc}
G_{0} & G_{-1} & G_{-2} \\
G_{1} & G_{0} & G_{-1} \\
G_{2} & G_{1} & G_{0}
\end{array}\right]=\left[\begin{array}{ccc}
\frac{G_{\max }}{2} & \frac{G_{\max }}{\pi} & 0 \\
\frac{G_{\max }}{\pi} & \frac{G_{\max }}{2} & \frac{G_{\max }}{\pi} \\
0 & \frac{G_{\max }}{\pi} & \frac{G_{\max }}{2}
\end{array}\right]
$$

In order to analyze the time-varying small-signal equivalent circuit of the two switching stage transistors shown in Fig. 2, Kirchoff's law can be utilized here. The gate-source voltage vector $\underline{V}_{g s I}$ and $\underline{V}_{g s 2}$ is expressed in terms of the voltage vector $\underline{V}_{s}$ at the common source point as

$$
\begin{aligned}
& \underline{V}_{g s 1}=-\left[\underline{\underline{I}}+\underline{\underline{G}}_{m 1} \underline{\underline{R}}_{s}+j \Omega \underline{\underline{C}}_{g s 1}\left(\underline{\underline{R}}_{i}+\underline{\underline{R}}_{s}\right)\right]^{-1} \underline{V}_{s} \\
& \underline{V}_{g s 2}=-\left[\underline{\underline{I}}+\underline{\underline{G}}_{m 2} \underline{\underline{R}}_{s}+j \Omega \underline{\underline{C}}_{g s 2}\left(\underline{\underline{R}}_{i}+\underline{\underline{R}}_{s}\right)\right]^{-1} \underline{V}_{s}
\end{aligned}
$$

where $\underline{I}$ is the identity matrix, $\underline{G}_{m l}$ is the conversion matrix for $g_{m 1}(t), \underline{\underline{G}}_{m 2}$ is the conversion matrix for $g_{m 1}(t), \underline{\underline{C}}_{g S 1}$ is the conversion matrix for $C_{g s 1}(t), \underline{C}_{g s 2}$ is the conversion matrix for $C_{g s 2}(t), \Omega$ is the diagonal matrix comprised by mixing frequency, $\underline{R}_{s}$ is the diagonal matrix with elements $R_{s}$, and $\underline{\underline{R}}_{i}$ is the diagonal matrix with elements $R_{i}$. The drain currents of transistors in switch quad can be expressed by the transconductance matrix and the voltage vector of $V_{g s}(t)$ :

$$
\begin{aligned}
& \underline{I}_{c 1}=\underline{\underline{G}}_{m 1} \underline{V}_{g s 1} \\
& \underline{I}_{c 2}=\underline{\underline{G}}_{m 2} \underline{V}_{g s 2}
\end{aligned}
$$

Considering the common source point using Kirchoff's current law, the tail current $\underline{I}_{s}$ can be expressed by

$$
\underline{I}_{s}=\underline{I}_{c 1}+\underline{I}_{c 2}+j \Omega \underline{\underline{C}}_{g s 1} \underline{V}_{g s 1}+j \Omega \underline{\underline{C}}_{g s 2} \underline{V}_{g s 2}-\frac{\underline{V}_{s}}{\underline{\underline{Z}}_{d s}}
$$

where $\underline{I}_{s}$ is the small-signal excitation vector. There is only one non-zero element in the current vector, which comes from the transconductance stage at RF frequency. Because the transistors at the transconductance stage are considered as linear time-invariant model, the currents at other harmonic frequencies are neglected.

Substituting (6), (7), (8) and (9) into (10), the current $\underline{I}_{s}$ can be expressed in terms of $\underline{V}_{S}$ as

$$
\begin{gathered}
\underline{I}_{s}=-\left[\left(\underline{\underline{G}}_{m 1}+j \Omega \underline{\underline{\underline{C}}}_{g s 1}\right)\left[\underline{\underline{I}}+\underline{\underline{G}}_{m 1} \underline{\underline{R}}_{s}+j \Omega \underline{\underline{\underline{C}}}_{g s 1}\left(\underline{\underline{R}}_{i}+\underline{\underline{R}}_{s}\right)\right]^{-1}+\right. \\
\left.\left(\underline{\underline{G}}_{m 2}+j \Omega \underline{\underline{\underline{C}}}_{g s 2}\right)\left[\underline{\underline{I}}+\underline{\underline{G}}_{m 2} \underline{\underline{\underline{R}}}+j \Omega \underline{\underline{C}}_{g s 2}\left(\underline{\underline{R}}_{i}+\underline{\underline{R}}_{s}\right)\right]^{-1}+\underline{\underline{Z}}_{d s}{ }^{-1}\right] \cdot \underline{V}_{s}
\end{gathered}
$$

The conversion gain of the switch quad $\left(G_{s w}\right)$ is defined as the ratio between the differential IF output voltage and the RF input current generated by the transconductance stage, which can be expressed by

$$
\underline{G}_{s w}=\frac{\underline{V}_{i f 2}-\underline{V}_{i f 1}}{\underline{I}_{s}}=\frac{\left(\underline{I}_{c 2}-\underline{I}_{c 1}\right)}{\underline{I}_{s}} \underline{\underline{R}}_{L}
$$

where $\underline{R}_{L}$ is the diagonal matrix with the load resistor. Combining (6), (7), (8), (9), (11) and (12) and setting $R_{s}$ to be zero, the $G_{S w}$ is derived as the below expression:

$$
G_{s w}=\frac{2}{\pi} \frac{A}{B+j C}
$$

where $\quad A=R_{d s} G_{\max } R_{L}$ 


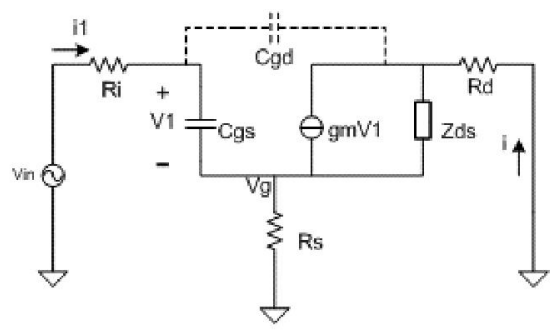

Fig. 3. The small-signal equivalent circuit for GaAs HMET

$$
\begin{aligned}
& B=1+R_{d s} G_{\max }-R_{d s} R_{i} C_{g s 0} C_{d s 0} \omega_{r f}^{2} \\
& C=\omega_{r f} C_{g s 0} R_{i}+\omega_{r f} C_{d s 0} R_{d s}+2 \omega_{r f} C_{g s 0} R_{d s}
\end{aligned}
$$

\section{B. Transconductance stage analysis}

Because the transconductance stage is excited by a small RF signal, the linear time-invariant analysis method can be utilized here. Fig. 3 shows the simple small-signal GaAs HEMT model used in the analysis. The $C_{g d}$ is not included to simplify the analysis process. The conversion gain of the transconductance stage $\left(G_{t r}\right)$ is the ratio between the RF current used to bias the switching quad and the input RF voltage. It is derived as:

$$
G_{r r}=\frac{g_{m-t} Z_{d s}-j \omega_{f f} C_{g s t r} R_{t r}}{R_{s_{-} t}\left(g_{m_{-} t} Z_{d s}-j \omega_{i f} C_{g_{-} t r} R_{s_{-} t r}\right)+\left[j \omega_{f} C_{g_{-} t r}\left(R_{i_{-} t r}+R_{s_{-} t r}\right)+1\right]\left(R_{s_{-} t r}+Z_{d s}\right)}
$$

where $Z_{d s}$ is composed of $C_{d s}$ and $R_{d s}$ in parallel and the parameters denoted by " tr" are the parasitic components in the transconductance stage. The overall conversion gain of the mixer $\left(G_{c n v}\right)$ is the product of $G_{s w}$ and $G_{t r}$, which is given as

$$
G_{c p v}=\frac{V_{i f 1}-V_{i f 2}}{V_{r f}}=\frac{V_{i f 1}-V_{i f 2}}{I_{s}} \frac{I_{s}}{V_{r f}}=G_{s w} G_{t r}
$$

\section{Simulation Verification}

The harmonic balance simulation is used to verify the analytical expression for mixer's conversion gain. The Angelov model [5] for GaAs HEMT is applied in the simulation. The parameters used in the theoretical calculation based on the analytical expression are also extracted from the Angelov model [5]-[7].

Two types of simulation conditions are setup. One is the fixed LO frequency condition, and the result is shown in Fig. 4. In this case, the full Angelov model is applied to the transistors in the transconductance stage and the simple Angelov model including $C_{d s}, C_{g d}, R_{d}$, and $R_{s}$, respectively, is applied to the transistors in the switch quad. The calculated conversion gain is almost identical with the simulated ones including $R_{d}$ and $R_{s}$. But it does not fit the curves including $C_{d s}$ or $C_{g d}$ well in high frequency range. The reason is that $C_{d s}$ and $C_{g d}$ in the switch quad are not included in the conversion matrix analysis, and the effect coming from these two parameters depends highly on frequency. Therefore, the theoretical expression for the conversion gain without $C_{d s}$ and $C_{g d}$ in the switch quad is not accurate enough at high frequencies. The fixed IF frequency

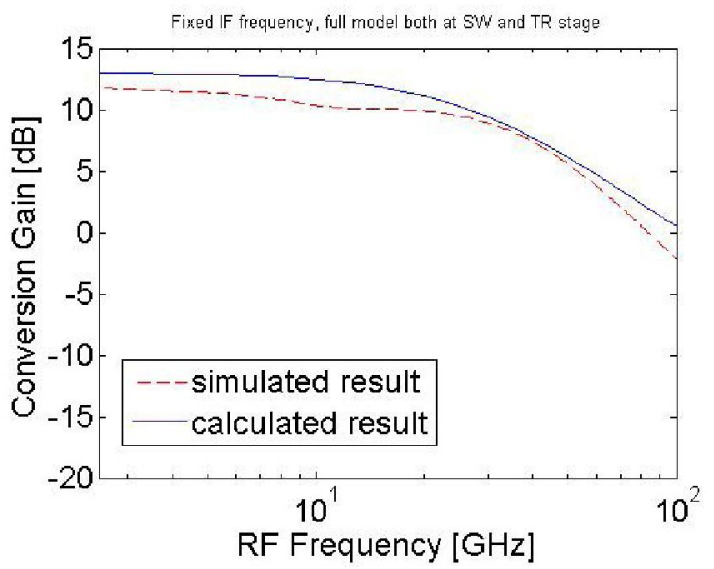

Fig. 5. Comparison of calculated and simulated active Gilbert cell mixer's conversion gain, IF frequency is fixed at $0.5 \mathrm{GHz}$, and $\mathrm{C}_{\mathrm{ds}}, \mathrm{C}_{\mathrm{gd}}, \mathrm{R}_{\mathrm{s}}$ and $\mathrm{R}_{\mathrm{d}}$ of the transistors in switching quad are all included in the simulation.

simulation is also implemented. The result is shown in Fig. 5.

\section{MEASUREMENT VERIFICATION}

The theoretical expression of the conversion gain is also verified by chip measurement experiment. A GaAs HEMT active Gilbert cell mixer using OMMIC ED02AH process is applied in the experiments. The measured conversion gains are calibrated simply by taking into account cable losses. The corresponding schematic of the measurement setup is simulated with Agilent ADS. The GaAs HEMT parameters of ED02AH process are also extracted from the ADS ED02AH transistor model for theoretical calculation.

Fixed LO frequency and fixed IF frequency conditions are setup for both chip measurement and ADS simulation. The measurement and simulation results are illustrated in Fig. 6 and Fig. 7 respectively. Although the measured conversion gain varies at high frequencies, the differences among measured, simulated, and theoretical calculated conversion gains are acceptable. The error mainly comes from the inaccuracy of the

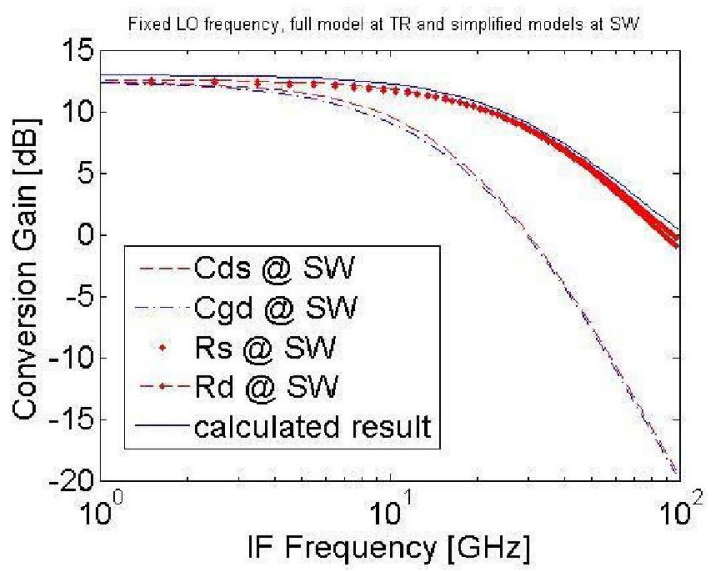

Fig. 4. Comparison of calculated and simulated active Gilbert cell mixer's conversion gain, $\mathrm{LO}$ frequency is fixed at $2 \mathrm{GHz}$, and $\mathrm{C}_{\mathrm{ds}}, \mathrm{C}_{\mathrm{gd}}$, $R_{s}$ and $R_{d}$ of the transistors in switching quad are included in the simulation one by one. 


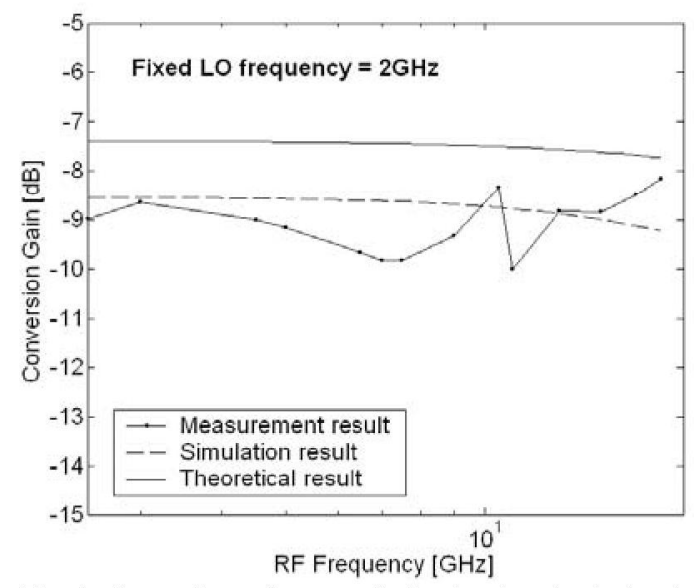

Fig. 6. Comparison of measured, simulated, and calculated active Gilbert cell mixer's conversion gain, LO frequency is fixed at $2 \mathrm{GHz}$.

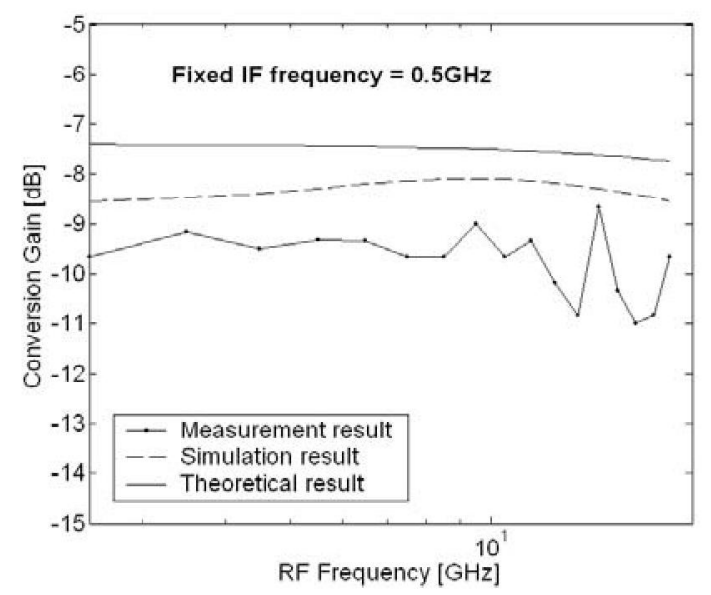

Fig. 7. Comparison of measured, simulated, and calculated active Gilbert cell mixer's conversion gain, IF frequency is fixed at $0.5 \mathrm{GHz}$.

ADS transistor model and nonlinear small signal circuit model of active Gilbert cell mixers.

\section{CONCLUSION}

The analytical expression for the GaAs HEMT active Gilbert cell mixer is derived by conversion matrix analysis when the transistor's model is simple enough. The expression is successfully verified by both ADS simulation and chip measurement. The excellent agreement is achieved between calculated and simulated results when the corresponding simple Angelov model is used in the simulation. It is also concluded that the mixer's conversion gain in high frequency domain can be increased by decreasing the effect originating from $C_{d s}$ and $C_{g d}$ of the transistors in the switch quad. Current effort concentrates on including a more complete description of the switch quad.

\section{REFERENCES}

[1] B. Gilbert, "A precise Four-quadrant Multiplier with subnanosecond Response," IEEE Journal of Solid-State Circuit, SC-3(4):365-373, Dec 1968
[2] S. A. Maas, Nonlinear Microwave and RF Circuits. Artech House, INC, 2. edition, 1988

[3] T. K. Johansen, J. Vidkjær, and V. Krozer, "Conversion Matrix Analysis of SiGe HBT Gilbert Cell Mixers", INMMC, 187-190, 2004 ROME.

[4] T. K. Johansen, J. Vidkjar, and V. Krozer, "Analysis and Design of Wide-band SiGe HBT Active Mixers," IEEE Transactions on Microwave Theory and Techniques, Vol. 53, No.7, July 2005

[5] Iltcho Angelov, Herbert Zirath and Niklas Rorsman, "A new Empirical Nonlinear Model for HEMT and MESFET Device," IEEE Transaction on Microwave Theory and Techniques, VOL.40, NO. 12, Dec. 1992

[6] Niklas Rorsman, Mikael Garcia, Christer Karlsson and Herbert Zirath, "Accurate Small-Signal Modeling of HFET's for Millimeter-Wave Applications," IEEE Transaction on Microwave Theory and Techniques, VOL.44, NO. 3, Dec. 1996

[7] Gilles Dambrine, Alain Cappy, Frederic Helodore and Edouard Playez, "A new Method for Determining the FET Small-Signal Equivalent Circuit," IEEE Transaction on Microwave Theory and Techniques, VOL.36, NO. 7, Dec. 1998 